\title{
La Shoah au miroir de la poésie dans Comme une chienne à la mort de Louise Cotnoir et Plus haut que les flammes de Louise Dupré
}

\section{Evelyne Ledoux-Beaugrand}

Université de Gand ${ }^{1}$

Le présent article se penche sur la référence structurante aux rafles et aux camps d'extermination dans les recueils de poésie de deux auteures québécoises: Comme une chienne à la mort de Louise Cotnoir (1987) et Plus haut que les flammes de Louise Dupré (2010). Les renvois au génocide des Juifs d'Europe dans la poésie de Cotnoir et Dupré participe moins d'un travail de pérennisation de la mémoire de la Shoah que de l'inscription de différentes expériences de la "précarité sociale" (Butler) dans un continuum.

This article examines the structuring reference to roundups and extermination camps in two poetry books by Québec authors: Louise Cotnoir's Comme une chienne à la mort (1987) and Louise Dupré's Plus haut que les flammes (2010). In Cotnoir and Dupré's poetry, the numerous mentions of the genocide of European Jews partake less to sustaining the memory of the Holocaust than to placing in a continuum different experience of "social precariousness" (Butler).

La Shoah est un ensemble d'événements de l'Histoire européenne désormais intégrés à une mémoire collective contemporaine dont les frontières s'étendent bien au-delà de l'Europe. Sous l'effet d'une mondialisation grandissante s'est produit depuis les années 1990 ce que Daniel Levy et Natan Sznaider désignent comme le découplage de la mémoire collective et de l'histoire nationale (89). Un regard porté sur la multiplication des sites commémoratifs hors du territoire européen et sur le traitement de ces événements dans des productions artistiques venues de pays non européens convainc du devenir cosmopolite de la mémoire de la Shoah. Dans le domaine littéraire, certains corpus semblent néanmoins plus propices que d'autres à la mobilisation de cette mémoire par les générations d'après pour des raisons pragmatiques de proximité géographique, historique, et culturelle ou parce que de nombreux survivants s'y sont installés après la libération des camps. Les écrits publiés en français au Québec ne se présentent pas d'emblée comme l'un de ces lieux privilégiés. Si l'exploration des legs mémoriels de la destruction des Juifs d'Europe donne lieu, notamment aux États-Unis et en France, à un nombre 
suffisamment important de récits et de romans pour que l'on puisse parler d'une littérature de la Shoah des deuxième et troisième générations, de telles catégories conviennent mal au contexte québécois francophone. Au-delà du nombre restreint d'œuvres littéraires ayant l'Holocauste pour trame de fond, il faut considérer le rapport filial aux victimes et aux survivants juifs du génocide que suggèrent fortement les catégories de deuxième et de troisième générations, bien qu'elles tendent de plus en plus à désigner un rapport de type affiliatif à ces événements. Or, pour des raisons relevant principalement du contexte socio-historique, la plupart des descendants des victimes du nazisme vivant au Québec ont l'anglais pour langue principale. ${ }^{2}$ Conséquemment, les références à la Shoah repérables dans les écrits de langue française sont plus rarement le fait des héritiers officiels de sa mémoire. ${ }^{3}$

Ni assez nombreux ni assez cohérents sur les plans thématique et formel pour constituer un corpus à part entière, les écrits publiés en français au Québec dans lesquels se repèrent des références à l'entreprise génocidaire nazie ne sont pas pour autant négligeables. Le travail pionnier et - malheureusement - laissé sans suite de Christine Poirier a permis de recenser, dans des récits, des romans et des œuvres poétiques parus dès $1950,{ }^{4}$ différents types d'allusions à la Shoah dont l'apport à la diégèse s'avère cependant souvent minime. Parus respectivement en 1987 et en 2010, les recueils de poésie Comme une chienne à la mort de Louise Cotnoir et Plus haut que les flammes de Louise Dupré font des rafles et des camps d'extermination une référence structurante, voire un miroir où se reflète une précarité humaine dont une certaine condition féminine serait l'un des visages.

Poirier propose les notions d'“échos" (2005) et de "récits obliques" (2008-2009) pour désigner l'affleurement ou le surgissement "par la bande (une ligne, un paragraphe, un poème)" de mentions de la Shoah ou des camps nazis “jusque dans les écrits de Québécois ni Juifs ni migrants qui ont pris connaissance des événements de manière indirecte” $(2008-2009,167)$. Cernant avec justesse un rapport allusionnel à ce pan funeste de l'histoire du vingtième siècle, ces 
notions se révèlent insatisfaisantes lorsqu'il s'agit de nommer le lien que tissent l'écriture des deux poètes entre les victimes du nazisme et les sujets féminins que sont la voix poétique de Plus haut que les flammes de Dupré et les présences féminines dans Comme une chienne à la mort de Cotnoir. ${ }^{5}$ Si les écrits des générations d'après traitant de l'extermination tendent à soulever des questions relatives à la transmission et pérennisation de la mémoire de ces événements ainsi qu'aux effets du genre testimonial, la référence à Auschwitz dans les œuvres de Dupré et de Cotnoir semble surtout appartenir au registre d'un savoir sur la vulnérabilité de l'existence et situer de ce fait les sujets féminins dans une généalogie de vies précaires. Le présent article examine comment, dans les recueils de Louise Dupré et de Louise Cotnoir, s'articulent ensemble la précarité des sujets féminins et les renvois à des nombreux événements historiques désormais rassemblés sous le nom singulier de Shoah afin de façonner un savoir sur ce qui se soustrait au voir. Leur poésie fait se regarder des histoires qui, a priori, ne se regardent pas, pas plus qu'elles ne semblent les regarder en tant qu'auteures québécoises, ${ }^{6}$ à moins d'adopter l'angle de vision particulier que suggèrent leurs écrits.

\section{Condition féminine sur fond de la Shoah}

Les réseaux de références au génocide des Juifs d'Europe et aux camps de la mort se dessinent de façons différentes dans Comme une chienne à la mort et Plus haut que les flammes, mais restent inextricables d'une prise de parole effectuée par un sujet féminin. Louise Dupré et Louise Cotnoir ont en commun une pratique d'écriture qui s'exprime avec constance depuis les années 1980, en particulier à travers la poésie, bien que l'une et l'autre s'adonnent également à la prose sous plusieurs formes. Toutes deux appartiennent de surcroit à une même génération de poètes québécoises “en majeure partie composée de femmes nées autour de 1949” (Brossard et Girouard 33) et venues à l'écriture au début des années 1980. Cette appartenance générationnelle ne serait qu'anecdotique si elle n'était pas liée, comme le soutiennent Nicole Brossard et Lisette Girouard 
dans L'Anthologie de la poésie des femmes au Québec, à "une mémoire d'avant le féminisme" (33) dont leurs écrits se font l'écho. Bien qu'un intervalle de treize années sépare la parution de la prose poétique de Louise Cotnoir dans Comme une chienne à la mort et les poèmes en vers libres de Louise Dupré composant Plus haut que les flammes, ces deux textes rendent manifeste le souci de leurs auteures de faire entendre la rencontre entre la voix singulière du sujet poétique et le nous collectif des femmes. Peut-être éloignée du formalisme pratiqué durant un temps par les poètes engagées dans le mouvement de libération des femmes, leur poésie noue toutefois l'intime à l'historique pour former une trame rappelant l'expression "Le privé est politique" qui a fait office de mot d'ordre de la production poétique des femmes durant les années 1970 alors qu'au Québec (tout comme en France) existait une très forte connivence entre militantisme et écriture.

Plus près dans le temps comme dans la forme d'une écriture au féminin clairement inscrite du côté d'une prise de parole collective, Comme une chienne à la mort est porté par un je difficilement identifiable. La voix poétique est en effet à la fois une et “plusieures," (1984) suivant le titre d'un précédent recueil de Cotnoir, et cette multiplicité affecte la lisibilité. Composé de quatre tableaux, eux-mêmes divisés en vingt courts textes, Comme une chienne à la mort suit dans chacune de ses parties le parcours d'une femme désignée uniquement par le pronom personnel elle. Cette femme semble être au nombre de quatre mais pourrait tout aussi bien n'être qu'une ou dix tant sont rares les marqueurs biographiques et socio-temporels qui permettraient d'établir une distinction entre les multiples facettes de son existence. “[C]hange[ant] sans cesse de référent" (Dupré 1995, 37) à l'intérieur d'un même tableau, elle est parfois une femme souffrant tantôt d'un cancer tantôt d'une forme de folie, alors qu'à d'autres occasions, elle est identifiée en tant que sans-abri ou survivante des camps nazis, ces dimensions s'additionnant plutôt que de s'annuler. Cette inconstance fait obstacle à la tentation d'y lire une histoire singulière et souligne les revendications à l'universalité portées par le elle: "Que cela empêche la datation et se pose bien au-delà d'une histoire particulière. Une sorte de polyphonie 
douloureuse où elle entend sa propre voix mêlée à celle du genre feminin” (Cotnoir 1987, 19). En se laissant "attein[dre] par ces bribes de récits racontés" (44) et venus d'ailleurs, elle brouille les traces du "singulier," qui selon elle "a quelque chose de honteux" (43).

Il est possible de lire l'instabilité identitaire des femmes de Comme une chienne à la mort à la lumière de leur interchangeabilité dans une économie patriarcale. Cet aspect est effectivement présent dans les poèmes de Cotnoir, en particulier dans le premier tableau où "l'usage" et la "généralité" des femmes sont rapprochés d'une forme d"“assassinat" (19). Mais ce manque d'assise est aussi un vecteur d'affiliations. Ces dernières se fondent, chez Cotnoir, sur la conscience d'une mortalité partagée, par ailleurs amplifiée par une position sociale de femme similaire par-delà le temps, l'espace, et les différences. Les sujets féminins aux frontières floues sont en effet liés à d'autres sujets vulnérables par le regard lucide qu'ils portent sur la mort et sur "la confusion du monde et son propre égarement," (46) ainsi que par une semblable précarité que surdéterminent les renvois à la déportation et aux camps de la mort. Écrits en prose, les poèmes de Comme une chienne à la mort ne sont pas pour autant narratifs. Dans ce recueil dont la trame demeure fuyante, il n'est pas surprenant que les renvois à la Shoah se donnent par bribes qui tissent ensemble un réseau nommant la persécution et l'extermination. Il s'agit, par exemple, de noms de lieux ou de termes associés aux ghettos et aux camps de la mort: "Elle se souvient de Figuren: à Vilna, les corps exhumés et brûlés," (33) “Cette femme refait la face du monde. Cracovie: la poésie des syllabes, l'insensé de l'histoire," "Le fantôme d'une petite inconnue suppliciée à Auschwitz" (84). Il est parfois plus directement question du parcours de la femme: "Elle en est revenue. Comme de ces camps de travail, d'extermination, de la mort" (16).

Plus haut que les flammes de Louise Dupré inscrit de façon plus manifeste la référence à Auschwitz, déjà suggérée dans les flammes du titre qui, rapidement, évoquent celles des crématoires. En revanche, la dimension collective de la voix poétique se fait moins prégnante. À la multiplicité du pronom elle, parfois accompagné chez Cotnoir d'une présence auctoriale 
surgissant dans des commentaires autoréflexifs sur l'écriture, Dupré préfère la deuxième personne du singulier. Ce tu clairement féminin se tient en équilibre à la jonction de l'intériorité et de l'extériorité, comme si le parcours singulier de la femme ne pouvait se dire que dans une certaine distance vis-à-vis d'elle-même. Se signale dans cette distance la portée plus générale de son expérience. La rencontre de la voix singulière et du nous collectif des femmes ne tient pas ici dans l'instabilité identitaire de la voix poétique et repose plutôt dans l'inscription évidente d'événements historiques funestes visant l'effacement radical de l'individualité, jusque dans la mort du sujet, ainsi que dans l'adresse à d'autres que suggère l'usage du $t u$ (même si celui-ci tient aussi lieu de je). C'est dire qu'un principe d'interchangeabilité des sujets féminins est aussi à l'œuvre dans Plus haut que les flammes et il opère à travers le recours à ce pronom personnel sans prédicat autre que son genre féminin et sa posture maternelle. Bien que singulier, le $t u$ s'arrime à d'autres existences que sont plus précisément des mères dont l'expérience, ici inextricable de la fragilité inhérente à l'existence humaine, se dévoile dans ce qu'elle a de plus intemporelle:

et le cœur explosé des mères depuis le jardin de la première femme

tu ne cesses de te demander comment marcher dans la douleur que tu traînes à ta semelle toi, l'exilée des grands vergers

en quête d'une réponse qui ne vient pas quel havre promettre à l'enfant près de toi (Dupré 70) 
Cette mère éternelle, dont le poème remonte la trace jusqu'aux "grottes/ où tu abritais tes petits," (20) reste indissociablement un fait de culture, comme l'indique l'évocation du mythe d'Ève dans les vers précédemment cités et des portraits de "madones/ et leurs larmes de marbre// versées sur des fils/ qui n'ont pas survécu/ à leurs bourreaux" (27).

Les quatre parties du recueil donnent à lire la traversée de l'obscurité qu'entreprend la voix poétique avant d'effectuer une remontée vers un univers de lumière et de danse, où persiste en contre-fond l'ombre d'un “monde à jamais endeuillé” (Dupré 101). Cette voix circonscrit d'emblée le lieu de son énonciation à l'espace du poème, qui est aussi l'espace où s'exhibe la vulnérabilité de l'existence, comme le laissent entendre les tout premiers vers de Plus haut que les flammes:

Ton poème a surgi de l'enfer un matin où les mots t'avaient trouvée inerte au milieu d'une phrase

un enfer d'images

fouillant la poussière des fourneaux

et les âmes sans recours réfugiées sous ton crâne

Aux côtés de la femme prend place un enfant à la santé fragile. La nature des liens entre elle et “cet enfant/ que tu n'attendais pas” (15) reste imprécise; c'est néanmoins une posture maternelle qu'adopte la voix poétique vis-à-vis de ce jeune garçon dont l'existence porte une promesse à la fois de vie et de mort, une innocence déjà grevée par son inéluctable perte que signalent les renvois nombreux à une piéta ainsi qu'à 'tous les minuscules/ vêtements d'Auschwitz/ et les biberons cassés" (69-70). Sa présence oblige la femme à se tenir dans un équilibre incertain au 
jointement de l'espoir et du désespoir, entre pulsion de vie et pulsion de mort, dans un espace saturé par la douleur.

\section{Un continuum de douleur}

Cotnoir et Dupré ne se contentent pas de mobiliser de manière ponctuelle les références à la Shoah. L'usage qu'elles en font se révèle plus extensif en ce qu'il inscrit l'horreur de l'extermination dans une continuité avec le quotidien, et ce, même dans Comme une chienne à la mort où les mentions de la persécution et de l'extermination par les Nazis apparaissent parfois sous la forme d'évocations qui exigent de la part des lecteurs et lectrices une connaissance relativement importante de l'histoire du génocide des Juifs d'Europe. Révélatrices d'une parenté secrète, de ce que Cotnoir nomme un ensemble de "raccords" (30) que seules les présences féminines de Comme une chienne à la mort arrivent à percevoir et à nommer, au prix d'être taxées de folles, les différentes évocations des camps de la mort font en effet office de point de jonction entre deux et même plusieurs déclinaisons de l'expérience de la précarité. Dans Plus haut que les flammes, l'espace du camp et l'espace domestique, surtout occupé par des femmes, basculent l'un dans l'autre à la faveur d'ambigüités sémantiques. Les chambres, les fours, et le rouge du sang comme celui des crayons de cire de l'enfant servent de connecteurs entre les expériences ainsi placées dans un même espace:

dans les chambres, les enfants vacillaient entre deux syllabes

collées

aux parois des bronches avant de s'écrouler

et les soldats tous les jours

la patience qu'il fallait

les fours à alimenter

les fours à nettoyer 
tu allumes le four

en tremblant

pour l'enfant près de toi

affamé comme un horizon

La poésie de Dupré poursuit son travail de connexion de l'espace intime et domestique avec l'histoire traumatique de la Shoah en des formulations parfois plus allusives: "la vie le soir/ est une lutte à finir/ dans la chaleur d'une chambre" (44). La chaleur dénoterait surtout les liens affectifs entre la femme et l'enfant si l'ambigüité de l'expression “lutte à finir" ne ramenait pas encore une fois le quotidien du côté de l'horreur en convoquant la chaleur de l'asphyxie dans les chambres à gaz et les fours crématoires.

Les poèmes mettent en place une sorte de présent éternel, une atemporalité dans laquelle les atrocités de la guerre s'imbriquent dans le quotidien des femmes, y demeurent en latence comme dans ce passage de Comme une chienne à la mort:

Les jours passent à ne pas se soucier du paradoxe. Elle néglige même de fredonner l'incantation propitiatoire. Elle oublie le mot victime avec le linge sur la corde. Elle va aux provisions, rédige quelques lettres, prépare les repas. Étonnée chaque fois que le massacre ait cette odeur de javel et de cuisine. (20)

C'est un continuum que la poésie de Dupré et de Cotnoir instaure entre la Shoah et les expériences féminines de folie, de maladie, d'itinérance, et de maternité inquiète et endeuillée, pour ne nommer que celles-ci. Il ne s'agit pas pour autant de rabattre l'une sur l'autre une condition féminine et une identité juive persécutée jusqu'à l'extermination. Pas plus que l'écriture poétique ne procède chez elles à une simple comparaison de ces deux registres de l'expérience de la vulnérabilité. Comparer suppose un hiatus, une séparation plus ou moins radicale entre les termes rapprochés à des fins d'analyse, même si dans le contexte propre à l'étude des représentations de l'extermination des Juifs d'Europe d'aucuns considèrent la comparaison comme une tentative condamnable de combler cet écart. À cet égard, Michael 
Rothberg note: “too often comparison is understood as 'equation' - the Holocaust cannot be compared to any other history, the story goes, because it is unlike them all" (23). Un point de vue auquel n'adhère pas Rothberg, on l'aura compris. Étendu à coup de "liens," d'“analogies" (Cotnoir 26), de "résonances," et d' "écho[s] dans la voix brisée" (41), l'espace de la rencontre de ces deux expériences est d'une autre teneur.

Ni comparatifs ni séparatifs, les rapports tissés par les deux poètes entre l'Holocauste et des expériences féminines et douloureuses du monde supposent d'emblée une filiation entre des événements de nature différente, pour ne pas dire inconciliable aux yeux de certains, ${ }^{7}$ sans que cela n'implique l'effacement des différences entre eux. Pour l'une des présences féminines de Comme une chienne à la mort, la comparaison ne peut qu'être boiteuse. Sa résistance à toutes formes d'analogies s'avère cependant vaine puisque les frontières entre l'horreur de l'extermination et sa propre histoire sont déjà abolies:

On n'imagine pas les hurlements sur les rives du fleuve Burg. ${ }^{8} \mathrm{C}$ 'est le silence, après, qui dérange. Il n'y a pas de texte pour le couvrir. Tout semble grave à cette femme mais il faut préciser ce qui l'accable. Elle risque: l'assentiment. [...] On ne peut pas inventer une telle horreur sans sombrer dans les limbes. Aussi n'y a-t-il plus de balises. Une forêt de conifères pousse sur les fosses en forme d'entonnoir. Comme si le monde était soudain frappé par un trou de mémoire. Elle ne veut pas y croire. Ne pas établir d'analogie entre son histoire et l'anéantissement. Elle hésite et boite quand elle compare. (Cotnoir 40, c'est moi qui souligne) Comparer tiendrait déjà d'une protection contre l'assentiment, c'est-à-dire contre la reconnaissance d'un espace partagé. L'absence de "balises" fait obstacle à une mise à distance qui serait, du moins dans ce fragment, salutaire à la voix poétique. Ailleurs dans Comme une chienne à la mort, la présence féminine s'emploie plutôt à faire reconnaître le continuum entre la condition des femmes et les victimes civiles de la guerre, que cristallise la figure de la survivante 
juive des camps nazis (et celles de mères éplorées, juives ou non juives, dans le recueil de Dupré):

Qui la prendra au sérieux? Elle s'acharne pourtant à montrer les liens, les analogies. Elle donne ses références, elle pointe les revues spécialisées, elle accumule les preuves. Rien n’y fait. L'hilarité générale quand la terre court à sa perte. Les femmes seules courbent l'échine et attendent la fin de l'hécatombe. Les autres jouent à la guerre perdue d'avance pour elles. [...] La guerre ne finit pas, elle ne commence pas, elle est. [...] Et les femmes mettent des couronnes de fleurs au cou des guerriers et sur la tombe du soldat inconnu. Elles entretiennent le décor, s’y posent en butin et refont la chair entre deux massacres. (Cotnoir 26)

La hantise du passé et la reconnaissance de la force de la vie qui poursuit malgré tout son œuvre sont des traits communs aux deux recueils. Ils s'illustrent notamment dans la manière dont "la mémoire des morts/ cherche une demeure" dans le présent (Dupré 104) et dans les "mères [qui] ont la foi" (28) mettent au monde des enfants condamnés à mort, ou par le biais d'images comme celle d'une "forêt de conifères [qui] pousse sur les fosses" (Cotnoir 40). Au-delà d'un travail de mémoire qui serait également un travail de deuil, les renvois à la Shoah dans les textes pointent l'enchevêtrement des existences soumises à la précarité. Si la violence, et la violence guerrière en particulier, tend à instaurer une frontière entre le Même et l'Autre, celui à protéger et celui à détruire, la connaissance partagée de la violence entraîne plutôt dans les poèmes de Cotnoir et de Dupré l'abolition de certaines frontières, en particulier des frontières nationales et temporelles.

\section{Généalogies de la précarité}

Dans Ce qui fait une vie, ${ }^{9}$ Judith Butler poursuit sa réflexion sur la "vie précaire" amorcée dans l'ouvrage éponyme traduit en français en $2005 .{ }^{10}$ Son travail sur la construction et les effets des catégories normatives d'humain et d'inhumain, c'est-à-dire la distinction entre une vie “jugée 
digne d'être pleurée" $(2005,46)$ et une vie dont la perte passe inaperçue, veut sortir d'une impasse de la pensée actuelle qui tend à mettre les "agendas [...] progressistes (féminisme, libertés sexuelles)" $(2010,32)$ au service de politiques guerrières ou visant à restreindre l'immigration (en particulier d'individus venant de pays islamiques). Pour contrer cette "fissure" $(2010,31)$ dans les mouvements sociaux, Butler propose de se concentrer "moins sur la politique identitaire, ou sur le type d'intérêt et de croyances formulés à partir des revendications identitaires, que sur la précarité et sa distribution différentielle” $(2010,36)$. Dans la reconnaissance de quelque chose de plus fondamental que les libertés individuelles, à savoir une précarité partagée et, surtout, la répartition inéquitable et organisée de cette précarité aux seins des populations et des groupes sociaux, Butler voit la condition essentielle du rapprochement de certains sujets pouvant, sur cette base, parvenir à former un “"nous” ténu” $(2005,46)$.

La précarité telle que l'envisage Butler n'est en rien naturelle. Ou plutôt, elle est d'abord naturelle, existentielle, "coextensive à la naissance elle-même” (2010 20) en sorte que toutes vies s'avèrent "précaires par définition: elles peuvent être effacées volontairement ou par accident; leur persistance n'est garantie en aucune manière" (2010, 30). La reconnaissance de la précarité de l'existence n'est cependant pas la même pour tous et se mesure à l'aune du deuil auquel se prêtent certaines vies. Un deuil qui va généralement de pair avec des politiques sociales assurant le maintien des vies "choisies," alors que d'autres existences ne bénéficient pas de ces conditions. La précarité existentielle, commune à "toute chose vivante" $(2010,23)$, se double donc d'une précarité sociale. Reconnaître que la vie est précaire, qu'elle peut à tout moment être perdue et qu'elle dépend toujours d'autrui, c'est assurer à cette vie les conditions sociales et relationnelles nécessaires non seulement à sa continuité, mais à sa qualité de "vie vivable" $(2010,27)$, à l'intérieur bien sûr des limites que lui impose son inéluctable finitude. En revanche, lorsque la précarité de certaines existences n’est pas appréhendée par les normes sociales, désignées en termes de cadres par Butler, ces êtres vivants sont alors relégués hors cadre, hors du champ des 
vies vivables, du côté des existences dont les souffrances et la perte passent inaperçues lorsqu'il ne s'agit pas, de façon plus radicale comme dans les contextes génocidaires, d'étiqueter d'emblée ces existences comme étant dispensables.

La réflexion de Butler sur la précarité, développée dans le contexte particulier de la guerre en Irak, se rattache à la question des renvois à la Shoah dans la poésie de Cotnoir et de Dupré là où Butler imagine une communauté "fondée sur la vulnérabilité et la perte” $(2005,46)$. Par là, il faut entendre la capacité de mettre en relation des êtres appartenant à des catégories identitaires que rien, a priori, ne rassemble, voire que tout éloigne, outre un semblable assujettissement à la violence de la précarité sociale, une même exposition "à son éventualité sinon à sa mise en œuvre” $(2005,46)$. "[L]a précarité met en évidence ce qui nous rend radicalement substituables et anonymes" $(2010,19)$ et l'interchangeabilité des présences féminines de Plus haut que les flammes et Comme une chienne à la mort est en effet inséparable de leur condition de femme. Une condition, il semble nécessaire de le rappeler, qui n'est en rien essentielle mais est au contraire "politiquement induite" et a pour effet d'exposer ces vies précaires "de façon différentielle à la blessure, à la violence et à la mort" (2010, 30). Le caractère substituable des femmes déjà repéré chez les deux poètes dénote leur vulnérabilité, tout comme la posture maternelle de la voix poétique du recueil de Dupré et les mentions d'une violence et d'une haine dirigées contre les femmes dans les poèmes de Cotnoir. La vulnérabilité qui les isole est également ce qui les lie non seulement à d'autres femmes, survivantes des camps ou mortes en déportation, et à des “mères/ au cœur éclaté” (Dupré 22) devant la destruction de leurs enfants, mais à tous ceux et celles qui ont connu un "destin de bétail” (Cotnoir 29).

Maintes fois énoncée autant chez Cotnoir que chez Dupré, la “douleur” que portent les présences féminines en raison de leur identité sociale de femmes agit comme un principe capable de rapprocher des existences éloignées dans le temps comme dans l'espace (géographique et culturel). Dans Plus haut que les femmes, elle est à la fois physique et psychique et tient entre 
autres dans le "regard/ de grande brulée" de la voix poétique qui revêt son "corps de douleur" (50). Cette voix est aussi bien habillée qu'habitée par cette sensation “installée/ dans tes cellules/ avec sa lumière froide" (65). "[T]rès vieille," (63) appartenant à des temps immémoriaux, sa douleur à partie liée avec des "mots [qui] laissent aussi/ des échardes/ sous la peau," (63) et avec le "silence/ des continents/ noyés" (63); partout elle accompagne la voix poétique. S'il est inséparable de son statut de femme, cet affect naît surtout de la combinaison de sa posture de mère et du savoir sur le monde qui en découle. Dans Comme une chienne à la mort, la douleur se noue inextricablement à l'identité de femme. Plus encore, femme est le nom même de sa douleur: "Il est si facile de mentir, glisse-t-elle, avec la certitude de saisir l'irréalisme de sa propre existence. Marquée au front, une douleur qu'elle nomme simplement femme. Lamento" (13). Plus qu'un mode d'existence, elle est un signe de reconnaissance que les présences féminines recherchent: "Elle est en quête d'un visage habité où l'œil serait barré par l'épreuve. Comme on dit: une grande douleur" (55).

Le passage suivant de Comme une chienne à la mort est significatif de la manière dont la douleur, en tant que synonyme de précarité, voire comme la conséquence de cette vulnérabilité sociale, est un "affect [...] doué [...] d'effet politique," (Butler 2010, 29) en ce sens qu'elle devient le vecteur d'une mémoire pour les oubliés:

Au mur, les livres d'Histoire. La Grande, l'Officielle. [...] Par choix, elle est amnésique. Elle ferme le paysage au paroxysme mortuaire, baisse les yeux. Il faut commencer par quelques désertions. Ne pas succomber à l'infamie. Cette femme se déguise, traverse beaucoup de siècles aux emblèmes douloureux. [...] Elle refuse l'anomie, signale toutes ces personnes qu'on a négligé d'aimer, dresse pour elles le Mur des Lamentations. Une sorte de protestation contre l'avilissement. (Cotnoir 72)

Si la douleur d'être femme la dépossède d'elle-même, lui laissant l'“envie irrépressible de visiter sa vie. Comme être à l'intérieur"' (Cotnoir 72), elle n'est pas pour autant un facteur d'isolement. 
De cette douleur, exprimée de façon plus manifeste dans le fragment suivant où sa bouche "se fige" et elle "trébuche" à l'évocation des "petits corps éteints, en cendres” (73), naît son amnésie choisie et sélective, celle-là même qui lui fait négliger la grande 1'Histoire au profit des anonymes auxquels n'ont pas été offertes les conditions assurant une vie vivable et dont la perte n’est pas été jugée digne d'être pleurée. "[S]'affiche[r] mortelle” (70), c'est dès lors s'inscrire dans un lignée d'êtres ayant en commun une vulnérabilité, un peu comme le fait la voix poétique de Plus haut que les flammes lorsqu'elle "continue [...] à visiter/ en silence/ les formes souffrantes" (Dupré 32) et devient “tout à coup/ l'artisane d'un linceul// pour les âmes/ sous les cendres" (80). Les généalogies de la précarité tissées par les deux poètes font jouer à l'envers le principe de substituabilité des vies précaires, ici mis au service d'une résistance à l'oubli qui va de pair avec la précarité sociale.

\section{L'angle de la honte: un savoir aveugle}

Les généalogies de la précarité déployées dans les recueils de Cotnoir et de Dupré rendent visibles des réseaux aux embranchements multiples (qui rappellent le schéma mémoriel multidirectionnel proposé par Rothberg). La poésie façonne tout autant qu'elle révèle des liens non pas tant secrets qu'invisibilisés ou inaperçus, ceux-là que rend visibles le voir aveugle auquel nous convient les textes de Dupré et de Cotnoir. Cela suppose de porter sur les vies précaires un regard "en diagonal," adoptant "l'angle de la honte," pour reprendre les termes dont use Louise Dupré dans les vers ouvrant la deuxième section de Plus haut que les flammes:

Et tu recommences ton poème avec la même main, le même monde, la même merde étalée sur la page

gauche, la main quand elle brasse des matières 
qui ne la regardent pas

sauf en diagonale

sous l'angle de la honte

L'angle de la honte diffère du regard honteux en ce qu'il est un point de vue, qui plus est un point de vue partagé et adopté à dessein et ne relève donc pas de l'incontrôlable affect qui submerge le sujet et l'écrase. Cet angle demeure cependant lié avec "l'angoisse du non humain" qui, selon Serge Tisseron, "borde toutes les formes de honte" (21).

Rassemblant un sentiment de grande vulnérabilité et un désir de se soustraire au "regard honnisseur de l'autre" (Tisseron 19), la honte est un affect éminemment social. En tant qu'“émotion de commande" (21) d'abord imposée de l'extérieur, elle fait l'objet d'un apprentissage inséparable d'un certain nombre de normes sociales tout en jouant un rôle désocialisant en ce que la honte procède à une forme de bannissement, à une exclusion de l'humanité. En empruntant à la terminologie de Butler, on peut dire que la honte menace le sujet d'une expulsion hors des cadres qui permettraient d'appréhender sa vie comme étant précaire et donc "dépend[ante] de réseaux de sociabilité" $(2010,29)$. La honte porte en elle la menace d'une expulsion hors de la catégorie d'humain. Tisseron avance pour preuve du pouvoir désocialisant de la honte certaines pratiques de la Grèce antique où la punition la plus grave ne consistait [...] pas dans la mise à mort, mais dans un bannissement à vie prononcé par un vote des citoyens qu'on appelait “l'ostracisme." Être banni signifiait être plongé dans la honte, et ses proches avec soi. Le banni était déclaré étranger dans son propre pays et devait prendre le chemin de l'exil. L'inverse est également vrai: être honteux, c'est éprouver l'angoisse de se sentir exclu du genre humain $(2006,21)$. 
Compris ici comme un regard jeté sur un sentiment d'exclusion de l'humanité et d'avilissement, l'angle de la honte est ce par quoi les voix poétiques approchent un savoir invisible car laissé hors cadre, pourrait-on dire en puisant encore une fois à même le lexique de Butler.

L'accès à cette connaissance suppose un regard autre qui tient en partie de la cécité. Pour la voix poétique de Comme une chienne à la mort, c'est par "cet obus dans mon œil qui fait tout sauter" (Cotnoir 14) que la vision s'ouvre à l'obscurité, elle-même identifiée en tant qu'envers du "mirage qui 1[a] guette" (17). La vision est aussi contrariée dans Plus haut que les flammes où l'aveuglement de la voix poétique n'entraîne pas un “rien voir;" il suscite au contraire une vision du "rien," de ce qui est négligeable et négligé:

c'était après ce voyage

dont tu étais revenue

les yeux brûlés vifs

de n'avoir rien vu

rien

sinon des restes $\quad(13-14)$

Les regards aveugles que porte sur le monde la poésie de Dupré et de Cotnoir se posent sur ce qui échappe à l'évidence. Les présences féminines s'abstraient d'un voir que l'on peut dire forcé afin de capter le rien des vies précaires vouées à une forme d'invisibilité. Les "yeux vides" (Dupré 51) de l'une et l'“iris brun" de l'autre, d'où surgissent "des images de sang, de feu. Des charniers. La part enfouie" (Cotnoir 57) deviennent le miroir permettant de regarder ce qui échappe en temps normal aux regards.

De cette forme d'aveuglement découle en effet la possibilité de voir des rapports que d'autres jugeraient fous: “Le triangle de sa vulve et l'Étoile de David. Il lui arrive d'être confuse. Mutilation, humiliation, emmurement. Elle énumère la kyrielle de l'atrocité comme les vieilles égrènent leur rosaire. Simple question de méthode. Il n’y a aucun rapport. Vraiment? Vous croyez? [...]" (Cotnoir 41). Si "[e]lle invoque la figure de Cassandre pour donner suite à son 
histoire" (37), sa parole n'est pas que vaines prophéties: elle "installe des lésions" (42) dans les apparences et instaure le doute chez ses interlocuteurs:

Il écrit qu'elle ne sait plus ce qu'elle dit. Schizophrène ou paranoïaque, il ne sait plus très bien lui-même à quoi s’en tenir. Parfois il se prend à douter. Elle si elle disait juste? Non.

Cela ne peut être. Mais cela questionne. [...] Maintenant, il entend le cri dans sa tête.

Non. La voix parasite sa fiction. (Cotnoir 30)

Qu'ils soient "brûlés vifs" (Dupré 13), "vides” (51), ou “crevés” (65) tels ceux d'Edipe à qui vient d'être révélée la face cachée de son histoire, les yeux de la voix poétique de Plus haut que les flammes sont frappés d'une forme de cécité qui les rend également doués de la capacité de voir les liens secrets, voire scandaleux entre la culture et la barbarie:

la vie est la vie

et l'on apprend à placer

Auschwitz ou Birkenau dans un vers (14)

Le regard honteux, celui que l'on détourne, ouvre le voir à ce qui se trouve derrière le champ du visible, “derrière la honte," là où “il y a aussi des mères/ au cœur éclaté// car le cœur n'est pas une abstraction” (22). Là où grouillent et vivent les vies précaires.

Le savoir auquel la voix poétique de Plus haut que les flammes accède par "cette fissure/ presque invisible/ dans la muraille du jour" (49) est précisément ce qui perce le champ du visible pour en montrer sa face obscure. S'y révèlent le savoir bas et sale de celle qui "appartien[t] à la terre, terreuse" (20), ainsi que la "banalité" et le "trivial" qui intéressent la présence féminine de Comme une chienne à la mort et l'amènent à arpenter un envers caché que Cotnoir désigne comme les “zones intermédiaires, souterraines” (57) de l'existence. Ce savoir tellurique et charnel, lié à la douleur d'être femme dans un monde qui juge certaines vies moins dignes que d'autres d'être soutenues et protégées, s'oppose dans les deux recueils à l'Histoire et aux formes nobles du savoir, représentées notamment par les papes et les récits hérités du catholicisme chez 
Dupré et par le il de Comme une chienne à la mort, sujet masculin capable de mettre à distance la souffrance du monde. Les expériences propres aux sujets féminins des recueils semblent en effet les rendre spécialement "récepti[fs] à ce qui ne se divulgue pas: les rafles, les déportations" (Cotnoir 91), ainsi qu'aux formes banales prises par l'horreur ici inscrite dans un rapport de continuité avec le quotidien des femmes:

ce qui reste d'Auschwitz
est un décor
de banlieue
petites maisons en brique
parfaite

comme en ces temps

d'anciennes naïvetés

avec des draps sur les cordes

balancés

à la moindre brise

et les femmes en tablier

sans taches

qu'elles exhibaient

devant leurs enfants (Dupré 30)

En retour, les références à la Shoah pointent une vulnérabilité humaine qui n'est pas circonscrite qu'aux horreurs de l'époque contemporaine. Auschwitz se présente comme une scène primitive sur laquelle se dévoile un savoir sur l'humanité tout entière, fait entre autres de "l'interminable liste/ des bûchers// allumés par des mains/ qu'on a dit humaines" (Dupré 67) et des "désastres" que sont les "pluies d'insectes/ ou de feu// pluies de pierre/ dont on lapide les épouses" (18). L'innocence est à jamais perdue et il semble désormais impossible aux présences féminines de se dérober à ce savoir qui les amène à jeter leur regard aveugle sur des périodes antérieures au génocide. "[C]e même théâtre primitif" (Cotnoir 41) se décline peut-être sous des jours et en des lieux différents, mais met toujours en scène des êtres dont la précarité existentielle, inhérente à toute vie, se double d'une précarité sociale. Autrement dit, si parenté il y 
a entre une certaine condition féminine et le destin réservé aux Juifs sous le régime nazi, cette filiation n'est pas le fait d'une faiblesse naturelle. Les expériences à travers lesquelles est rendue visible la vulnérabilité sociale de certains sujets s'inscrivent certes dans une sorte de simultanéité atemporelle mais n'en sont pas moins partie intégrante de l'Histoire. C'est l'Histoire qui place ces sujets du côté de la honte, d'un avilissement qu'entraîne l'absence de mesure venant assurer à une vie le soutien et les conditions nécessaires à sa viabilité (à l'intérieur des limites de son inéluctable finitude), comme le rappelle Louise Cotnoir: "Le cours sinueux de l'Histoire la rend aisément vulnérable" (73).

Que des poètes québécoises convoquent des références à l'Holocauste n'a en soi rien de surprenant à l'heure où la mémoire du génocide des Juifs d'Europe s'impose en tant que paradigme mémoriel: "the Holocaust has become a template for collective memory in areas of the world that had nothing to do with those events but that have known other collective traumas" (Suleiman 2). Or, davantage que la mémoire liée en propre aux événements historiques désormais désignés du nom singulier de Shoah, c'est un savoir plus archaïque sur la précarité que signalent les références au programme nazi d'extermination qui structurent la poésie de Louise Dupré et de Louise Cotnoir dans Plus haut que les femmes et Comme une chienne à la mort. Tout en occupant une place de choix dans les poèmes, la Shoah se présente en effet comme l'un des chapitres d'une histoire humaine composée de "tous ces récits/ de sang et de couteaux// depuis les grottes/ où tu abritais tes petits" (Dupré 26). Un travail de (contre-)mémoire s'accomplit effectivement dans ces textes où les voix poétiques percent dans des pans de l'Histoire et de la grande culture des trous par lesquels l'on peut apercevoir des existences dont les souffrances restent inaperçues des normes sociales, mais la désignation de trauma collectif s’applique mal aux différentes expériences de la précarité explorées par Dupré et Cotnoir. Le trauma collectif implique il est vrai 
une certaine synchronie qui s'avère inconciliable avec l'atemporalité caractéristique des généalogies de la précarité déployées dans ces deux recueils.

S’il y a effectivement résonances entre le trauma du génocide et la condition féminine, c'est dans la posture de vulnérabilité engendrée par les contingences historiques que celles-ci se font entendre. Une certaine condition des femmes, dont les racines puisent à même des temps immémoriaux, place certes les présences féminines des deux recueils au plus près des fondements de l'humanité, de ce que d'aucuns considèrent comme ses bas-fonds, là où se révèle toute la vulnérabilité de "l'animal humain" (Butler 2010,23) que la culture cherche au mieux à pallier par des mesures sociales et politiques, au pire à dénier chez des sujets qu'elle se refuse de considérer. La référence structurante à l'histoire du génocide perpétré par les Nazis nous rappelle toutefois que cette précarité n'est pas une condition naturelle échouant à certaines catégories de sujets (les femmes, les minorités). Peut-être intime et vécue isolément, la vulnérabilité a toujours partie liée avec le social et le politique. Sous le signe d'un continuum entre les registres du privé et du politique, l'écriture de Dupré et de Cotnoir entretisse différentes histoires de douleur, montrant ainsi comment elles sont amenées à se faire le miroir l'une de l'autre alors que d'emblée rien ne semble les vouer à se réfléchir. Leur poésie nous pousse du même coup à réfléchir à la façon dont ces histoires s'imbriquent dans un même espace qu'est celui de la vulnérabilité sociale. Si l'on peut dire que Comme une chienne à la mort et Plus haut que les flammes envisagent la Shoah sous l'angle d'une condition féminine soumise à la précarité, l'inverse est aussi vrai: la condition féminine y est examinée à l'aune de la Shoah. Inscrites dans un rapport spéculaire par la poésie de Dupré et de Cotnoir, la Shoah et des expériences féminines marquées par la fragilité et (la menace de) la perte se font le reflet d'un savoir qui donne à voir cette part d'invisibilité que sont les vies dont la précarité n'est pas appréhendée par les cadres sociaux.

\section{Notes}




\section{Ouvrages consultés}

Brossard, Nicole, et Lisette Girouard. Anthologie de la poésie des femmes au Québec: des origines à nos jours. Montréal: Remue-ménage, 2003.

Butler, Judith. Vie précaire. Les pouvoirs du deuil et de la violence après le 11 septembre 2001. Paris: Amsterdam, 2005 [2004].

---. Ce qui fait une vie. Essai sur la violence, la guerre et le deuil. Paris: La Découverte, 2010 [2009].

Cotnoir, Louise. Plusieures. Trois-Rivières: Écrits des forges, 1984.

---. Comme une chienne à la mort. Montréal: Remue-ménage, 1987.

---. Signature païenne. Montréal: Remue-ménage, 1989.

Dupré, Louise. "Louise Cotnoir: le romanesque de la poésie en prose.” Tangence 47 (1995): 3441.

---. Plus haut que les flammes. Montréal: Noroît, 2010.

Leblanc, Carl. Artéfact. Montréal: XYZ, 2012.

Levy, Daniel, et Natan Snaider. "Memory Unbound: The Holocaust and the Formation of Cosmopolitan Memory.” European Journal of Social Theory 5.1 (2002): 87-106.

Mavrikakis, Catherine. Le ciel de Bay City. Montréal: Héliotrope, 2008.

Molin Vasseur, Annie. "Louise Cotnoir: Un appel à la lumière.” Lettres québécoises 138 (2010): 6-9.

Poirier, Christine. "La Shoah dans la littérature québécoise de langue française.” Mémoire de maîtrise, Département de langue et littérature françaises, Université McGill, 2004.

---. "Échos de la Shoah dans l'œuvre poétique de Jacques Brault, Irving Layton et Leonard

Cohen." Voix et images 30.3 (2005): 43-55.

---. "Récits obliques de la Shoah dans le roman québécois de 1945 à 1980." Canadian Jewish Studies/Études juives canadiennes 16-17 (2008-2009): 167-184.

Ringuet, Chantal. Le Sang des ruines. Gatineau (Québec): Écrits des Hautes Terres, 2010.

Rothberg Michael. Multidirectional Memory: Remembering the Holocaust in the Age of Decolonization. Stanford: Stanford University Press, 2009.

Shvets, Catherine. Hitler et la fillette. Montréal: Flammarion, 2010.

Suleiman, Susan Rubin. Crisis of Memory and the Second World War. Cambridge and London: Harvard University Press, 2006.

Tisseron, Serge. "De la honte qui tue à la honte qui sauve.” Le Coq-héron 1.184 (2006): 18-31.

Vaugeois, Denis. “Les juifs, la langue et l'école.” Le Devoir, 26 février 1999 [en ligne] http://archives.vigile.net/pol/minorite/vaugeoisjuifs.html 
${ }^{1}$ Cet article s'inscrit dans le cadre d'un projet postdoctoral financé par le Conseil de recherches en sciences humaines du Canada.

${ }^{2}$ Sous le régime français, les Juifs, au même titre que les Protestants français (huguenots), se voyaient refuser le droit de s'établir en Nouvelle-France à moins de consentir à se convertir au catholicisme. L'installation des premiers représentants de la communauté juive sur le territoire de ce qui deviendra le Québec n'a lieu que sous le régime britannique et la plupart d'entre eux arrivent alors de New York, où se trouve déjà une importante communauté avec laquelle ils maintiennent des liens forts (Vaugeois). Ils adoptent la langue officielle du moment: l'anglais. L'importante vague d'immigrants juifs que connaît le Québec à la fin du dix-neuvième siècle est principalement composée d'hommes et de femmes venus d'Europe de l'Est. Allophones, ils s'intègrent à la communauté anglophone déjà établie. Ce n'est qu'au courant des années 1950, après les Indépendances des pays du Maghreb, que des Juifs sépharades, dont plusieurs sont francophones, choisissent d'immigrer au Québec.

${ }^{3}$ Avec des exceptions notables, dont Régine Robin.

${ }^{4}$ Aussi bien en langues française qu'anglaise.

${ }^{5}$ Cet apport plus structurant se remarque dans quelques œuvres d'auteurs juifs ou non juifs qui font de la référence à la Shoah et à sa mémoire leur principal pivot. Pensons entre autres aux poèmes de Chantal Ringuet composant Le Sang des ruines, aux romans Le ciel de Bay City de Catherine Mavrikakis, et Artéfact de Carl Leblanc, et aux nouvelles de Catherine Shvet rassemblées sous le titre Hitler et la fillette.

6 Il faut cependant préciser que la grand-mère paternelle de Cotnoir était juive (Molin Vasseur 8). Dans l'entrevue donnée à Annie Molin Vasseur, l'auteure ne révèle cependant pas si elle était une survivante de la Shoah. Signature païenne, un recueil en prose poétique paru en 1989, en dit plus sur cette grand-mère paternelle d'origine juive allemande. Désignée tout au long de Signature païenne par son nom en partie tronqué, "L. Sigman" (10), scandé à plusieurs reprises ou "Sigman, la dernière du nom" (66), "l'Aïeule" (44) dont le prénom, "Laetitia" (89), n'apparaît qu'une fois en entier, est sommairement identifiée en tant que survivante du génocide des Juifs d'Europe. La voix poétique avance à tâtons vers les images de cette femme qu'elle n'a pas ou peu connue, à qui elle "invente" (43) une vie où désir, plaisir, et amour dominent le malheur. Les références à l'Histoire sont quasi inexistantes dans ce recueil qui fait la part large à la question de la filiation: "Une ombre portée sur son histoire" (25).

${ }^{7}$ En est un bon exemple la polémique autour du poème "Lady Lazarus" dans lequel Sylvia Plath campe le désir de mourir du sujet poétique sur fond des atrocités commises par les Nazis, les mentions aux traitements inhumains contre les Juifs servant surtout à signifier sa souffrance et son sentiment d'impuissance.

${ }^{8}$ La rivière Burg évoque le camp d'extermination de Sobibor, situé sur cette rivière de Pologne, à proximité de l'Ukraine.

${ }^{9}$ Dans la version originale anglaise, parue en 2009 chez Verso, l'ouvrage s'intitule Frames of War: When is Life Grievable? 
${ }^{10}$ Originalement publié en anglais par Verso, en 2004, sous le titre: Precarious Life: The Powers of Mourning and Violence. 